\title{
Is pelvic-floor morphology a predictor of successful pessary retention? Original research and review of the literature
}

\author{
F S Paterson, ${ }^{1}$ Bsc Hons, MB BCh, MMEd (O\&G), FCOG (SA); Z Abdool, MB ChB, MMed (O\&G), FCOG (SA)
}

\author{
${ }^{1}$ Department of Urogynaecology, Obstetrics and Gynaecology, Faculty of Health Sciences, Steve Biko Academic Hospital, University of Pretoria, \\ South Africa
}

Corresponding author: F S Paterson (drfpaterson@gmail.com)

Background. Vaginal pessaries are known to be an effective treatment modality for pelvic organ prolapse (POP). Pessaries form an important part of the physician's armamentarium in the treatment of POP, but currently many of the factors affecting their successful use are poorly understood.

Objectives. To determine the association between pessary retention (PR) at 1 year, and functional pelvic floor morphology, i.e. levator hiatal distance and area, and levator avulsion.

Methods. This retrospective study reviewed the records of 73 patients with symptomatic POP at a tertiary urogynaecological centre. This multi-ethnic cohort had previously been studied for pelvic-floor morphology, had had 4D transperineal pelvic-floor ultrasound, and had opted for a vaginal pessary as a treatment option.

Results. Our population had a mean age of 59.4 (range 32 - 91) years, and mean body mass index of 29.4 (range 20 - 42) $\mathrm{kg} / \mathrm{m}^{2}$, with a mean assessment of stage 3 in the Pelvic Organ Prolapse Quantifications System (POP-Q). The level of prolapse was found to be related to PR $(p=0.077)$. We further explored this concept using symmetric measures of association $(\gamma=-0.353)$, indicating that PR decreases with increasing prolapse severity. PR was also found to be inversely associated with prior pelvic reconstructive surgery $(n=63 ; p=0.055$; $\gamma=-0.417)$. There was a strong correlation that failed, however, to achieve significance by a small margin $(p=0.052)$ between hiatal distance on contraction and PR.

Conclusion. This study found an inverse relationship between PR and hiatal distance on contraction, prior pelvic surgery and the severity of prolapse. This was a pilot study with a limited number of participants, and the authors plan a prospective study to further clarify the association between long-term PR and functional pelvic floor morphology.

S Afr J Obstet Gynae 2018;24(3): 74-78. DOI:10.7196/SAJOG.2018.v24i3.1360

Pelvic organ prolapse (POP) is a common, chronic pelvic-floor disorder. The epidemiology of POP in western society is well defined, but in developing countries, and particularly in South Africa (SA), data on the true prevalence are scanty. ${ }^{[1]}$

In the western world, it is estimated that approximately $10 \%$ of women will have surgery for POP at some stage in their lifetime, and of those, a third will have further operations for POP ${ }^{[2]}$ In the USA, POP constitutes the most common reason for hysterectomy in menopausal women. ${ }^{[3]}$ One of the largest studies on the epidemiology of POP found that women have a lifetime risk of 2.04 per 1000 person-years of developing POP. ${ }^{[4]}$ Interestingly, in one of the few available SA studies, Naylor ${ }^{[5]}$ found that out of 2091 hysterectomies performed, $29 \%$ were performed for prolapse.

Research into POP is driven by the fact that it has a negative effect on patient quality of life, may lead to a general health decline, which includes increased risk for falls, and may thus lead to major morbidity. ${ }^{[6]}$ Therefore, POP and concomitant urological issues are of significance to the individual in terms of their effect on morbidity and quality of life. They are equally of importance to society, in view of the burden on economic and other resources necessary for treatment of the disease.

Treatment for POP can be divided into the conservative and the surgical. Conservative management includes pelvic-floor therapy and the use of vaginal pessaries. ${ }^{[1,2]}$ Surgical management can be performed through either the abdominal or the vaginal route. ${ }^{[7]}$ Geoffrion et al. ${ }^{[8]}$ found that approximately $67 \%$ of women will select conservative management as primary management of the condition. In SA, the usage of vaginal pessaries by patients may be far lower, possibly as a result of lower uptake by local gynaecologists. In a study on the use of vaginal pessaries by SA gynaecologists, Abdool ${ }^{[9]}$ found that only a quarter felt comfortable offering their patients vaginal pessaries for POP.

Previous studies on this matter have been limited by an absence of standardised terminology pertaining to successful PR. As Mutone et al. ${ }^{[10]}$ clearly state, 'success is an evolving, time-dependent concept.' To better understand the factors affecting pessary retention (PR), the length of time for which the pessary was successful needs to be considered. For the purposes of this study, we have made distinctions between initial pessary fitting, short-term PR and long-term PR. This is necessitated by the fact that different factors affect the success of pessary use at different times. This is clinically relevant for physicians, as knowledge of these factors allows one to determine the factors associated with short- and long-term pessary use.

Success of initial pessary fitting: There is conflicting evidence as to the factors affecting the initial fitting success. Clemons et al. ${ }^{[1]}$ found that out of 100 patients fitted with a pessary, no single patient 
demographic or comorbid condition was associated with pessary success or failure. Maito et al. ${ }^{[12]}$ found mild posterior prolapse to be an indicator of initial fitting success, and that age, body mass index (BMI), parity, pelvic resting tone and Oxford score, prior incontinence and comorbidities all had no effect on pessary fitting success. In contrast to this, other studies have found that younger age, obesity and poor pelvic-floor muscle tone are all associated with initial fitting failure. ${ }^{[13]}$ Two studies found that the prolapse Pelvic Organ Prolapse Quantifications System (POP-Q) stage had little effect on initial pessary fitting, and therefore the authors advocate conservative management with vaginal pessaries as a treatment modality for prolapse, regardless of severity. ${ }^{[9,12]}$ Fig. 1 illustrates stage 3 anterior compartment prolapse.

There is little data available with regard to prior surgery and pessary success. In one of the first studies to examine the success of PR after hysterectomy or previous pelvic reconstructive surgery for POP, Nemeth et al. ${ }^{[14]}$ found that one-fifth of the patients who had had previous reconstructive surgeries had unsuccessful initial fittings due to changes in their vaginal anatomy. This included vaginal shortening $(<4 \mathrm{~cm})$ and increase in vaginal width $(>3$ finger breadths). Their findings indicated that following one pelvic reconstructive surgery, the likelihood of failure was $18.7 \%$. After three or more operations, the risk of failure was $\sim$ one in every three cases.

Other studies have also found a statistically significant relationship between success of the initial fitting and previous hysterectomy and/or surgery for POP. ${ }^{[9,12]}$ Clemons et al. ${ }^{[15]}$ found that factors affecting unsuccessful pessary trial included a short and/or wide vaginal introitus. Their findings indicated

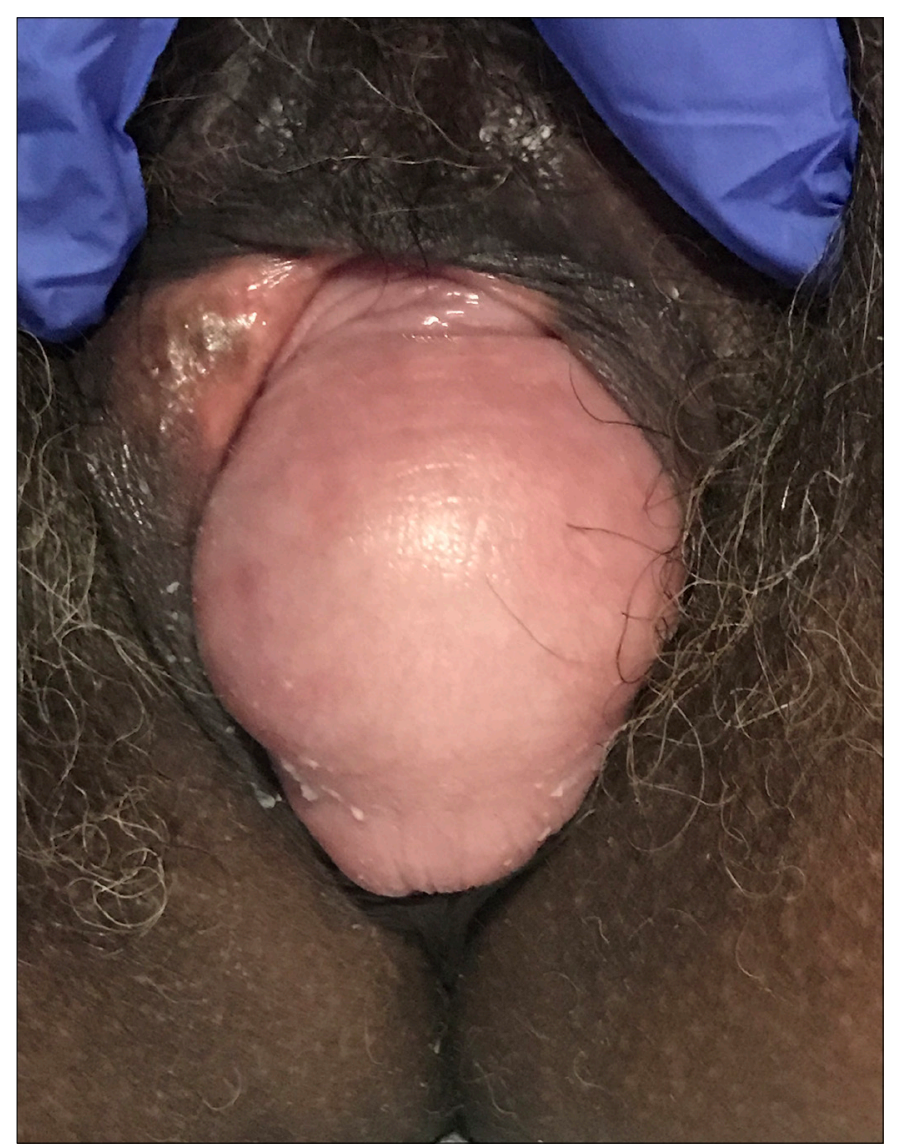

Fig. 1. Pelvic Organ Prolapse Quantifications System (POP-Q) stage 3 anterior compartment prolapse. that successful fitting is associated with total vaginal length $(>6 \mathrm{~cm})$, and size of the vaginal introitus ( $<4$ finger breadths).

Short- to long-term PR: Geoffrion et al. ${ }^{[8]}$ state that younger age at presentation ( $<65$ years), smoking, a genital hiatus/transvaginal length of $>0.8$, and lower overall POP-Q score all decrease the likelihood of short-term use. Age $\geq 65$ years is one of the strongest predictors of continued, long-term use of pessaries. ${ }^{[12]}$ Additionally, patient desire to either undergo or to avoid surgery, severe posterior compartment prolapse, apical prolapse, large genital hiatus, as well as advanced prolapse (POP-Q stage 3 and 4 ) are all associated with decreased likelihood of long-term ( $>1$ year) PR. ${ }^{[12,16]}$

In one of the only published trials that has looked at levator morphology and PR, Cheung et al. ${ }^{[17]}$ reviewed the data of 255 women who had retained a vaginal pessary for $\geq 1$ year. They found that pessary expulsion within a year was more likely in women with advanced prolapse. They found that pessary expulsion within a year was more likely in women with advanced prolapse. Their main findings in terms of levator morphology were that women with larger hiatal dimensions both at rest and on valsalva, larger hiatal areas at rest, contraction and valsalva as well as levator ani muscle avulsion were less likely to retain a pessary in the long term $(>1$ year). It is self-evident that levator avulsion is associated with more advanced prolapse, but after controlling for potential confounders, levator muscle avulsion had a persisting impact on pessary expulsion (odds ratio $3.18 ; p<0.01$ ).

Research has yet to enable a complete understanding of the pathophysiology of POP. However, avulsion or macrotrauma of the levator (defined as disconnection of the puborectalis muscle from its insertion on the inferior ramus and pubis), and microtrauma causing pathological overdistension of the levator are thought to play a role in some types of POP. ${ }^{[18.19]}$ There is currently scant data describing the association between levator morphology and successful pessary use. Studies examining factors affecting PR mainly focus on attributes that can be ascertained by clinical examination alone. However, imaging of the pelvic floor via ultrasound is becoming a more accessible method of evaluation, and is gaining traction in the gynaecological community. A study of the association between PR and functional pelvic-floor muscle morphology will add to the decision-making paradigm between clinician and patient.

The aim of our study was therefore to determine if altered levator ani muscle morphology (in the form of distension and avulsion) is a contributing factor to successful pessary use. The expectation is that the discovery of standardised objective parameters that affect the success of pessary use will allow for better patient selection, and ultimately lead to more effective pessary use.

\section{Objective}

The objective this study was to evaluate the association between $\mathrm{PR}$, demographic factors and functional anatomy of the pelvic floor, i.e. levator hiatal distance and area and levator distension, using $4 \mathrm{D}$ ultrasound.

\section{Methods}

This is an analytic, retrospective cross-sectional study involving women seen at a urogynaecology clinic between 2012 and 2015. This multi-ethnic cohort had previously been studied for pelvic floor morphology, had had 4D transperineal pelvic floor ultrasound, and had opted for a vaginal pessary as a treatment option. ${ }^{[19]}$ We 
retrospectively analysed 74 data sets of women referred for symptomatic pelvic organ prolapse, as defined by the Validated International Consultation on Incontinence - Vaginal Symptoms questionnaire. ${ }^{[19]}$ After gaining informed consent and taking a detailed history, clinical assessment using the International Continence Society POP-Q for prolapse grading was performed.

Following bladder-emptying, a 4D transperineal ultrasound was performed, with the woman in the supine position (Fig. 2). Volumes were acquired at rest, maximum pelvic-floor contraction, and maximum valsalva manoeuvre using a Voluson $i$ (GE Health, USA) ultrasound system with a RAB4-8 $\mathrm{MHz}$ probe. Levator hiatal area measurements and levator avulsion were analysed offline 6 - 8 weeks later, using 4D View software (GE Kretztechnik, Austria). Levator avulsion is defined as a traumatic

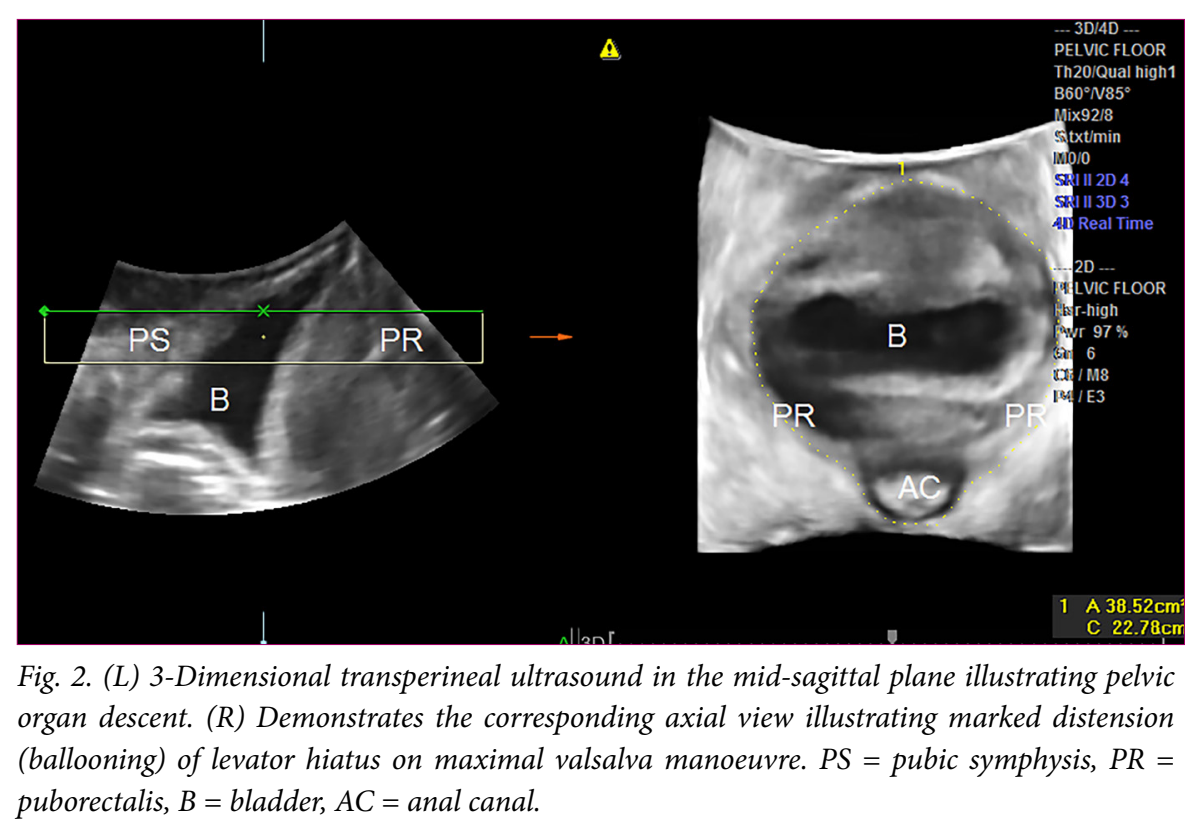

Fig. 2. (L) 3-Dimensional transperineal ultrasound in the mid-sagittal plane illustrating pelvic organ descent. (R) Demonstrates the corresponding axial view illustrating marked distension (ballooning) of levator hiatus on maximal valsalva manoeuvre. $P S=$ pubic symphysis, $P R=$ puborectalis, $B=$ bladder, $A C=$ anal canal .

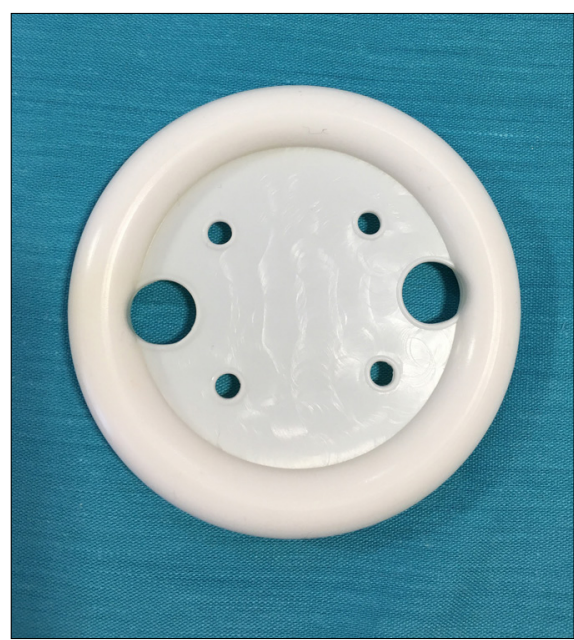

Fig. 3. An example of a typical ring pessary with support. disruption of the puborectalis muscle from its insertion point on the pubic bone. ${ }^{[18]}$

Pelvic muscle tone was assessed per vaginam using the Oxford grading scale, where participants are asked to contract their pelvic musculature (squeeze examiner's finger, which has been gently inserted into the vaginal introitus) as strongly as possible and then to relax. The Oxford system uses a five-point grading scale where 0 denotes no contraction, 1 a flicker of movement of the pelvic musculature, 2 a weak contraction, and 3, 4 and 5 a moderate, good and strong contraction, respectively. ${ }^{[19]}$

In our unit, the preferred first-line pessary is a ring with support (Fig. 3). Following insertion, the patients are reviewed after 1 month, and thereafter at 3-monthly intervals. Short-term success was defined for the purpose of this study as $<6$ months, and long-term success as retention for $\geq 1$ year.
Data were captured using Excel (Microsoft, USA). Statistical analysis was performed using SPSS version 25 (IBM Corp., USA) statistical software. Descriptive statistics were utilised for demographic data, and Pearson's $\chi^{2}$ test to determine the association between maximum PR and patient factors such as age, BMI, parity, levator avulsion and previous surgery. Analysis of variance was used to analyse the association between $\mathrm{PR}$ and parity, as well as ultrasonographic measures of functional anatomy, namely levator hiatal distance, levator hiatal area (distension) and levator avulsion. $P<0.05$ was used to indicate statistical significance.

\section{Results}

Of the 74 patients in our dataset, complete demographic and ultrasound information was available for 73 . The mean age was 59.4 (range 32 - 91) years. Twenty-two percent $(n=14)$ patients reported a previous hysterectomy, while $63(85 \%)$ patients had undergone some form of previous surgery for prolapse (including anterior repair, posterior repair, sacrospinous fixation, abdominal sacrocolpopexy, or a combination thereof).

Table 1 demonstrates the association between demographic characteristics and risk factors for PR.

Combining the six surgical categories above to form a category of 'any previous pelvic surgery', we found an association with PR $(p=0.055)$. Using symmetrical measures of association, we found a $\gamma$-value of -0.417 , indicating an inverse relationship between PR and previous pelvic surgery.

The average prolapse, as per the POP-Q staging system, was 2.53. Thirty-nine percent $(n=29)$ had major prolapse (stage 3 or 4 ) according to POP-Q system. The

Table 1. Demographic data $(N=73)$

\begin{tabular}{|c|c|c|}
\hline Parameter & Value, mean/frequency & $p$-value* \\
\hline Age, mean (SD) & $59.40(12.78)$ & 0.195 \\
\hline Parity, median (range) & $4.00(0-9)$ & 0.70 \\
\hline Previous hysterectomy, $n$ (\%) & $14(22)$ & $\mathrm{na}^{\dagger}$ \\
\hline Previous prolapse surgery, $n(\%)$ & $63(85)$ & 0.055 \\
\hline Oxford resting tone & 2.33 & 0.713 \\
\hline POP-Q staging & 2.53 & 0.077 \\
\hline Levator hiatal distance (rest) & 42.40 & 0.228 \\
\hline Levator hiatal distance (contraction) & 33.20 & 0.052 \\
\hline Levator hiatal distance (valsalva) & 59.24 & 0.207 \\
\hline Levator hiatal area (rest) & 26.27 & 0.674 \\
\hline Levator hiatal area (contraction) & 21.45 & 0.950 \\
\hline Levator hiatal area (valsalva) & 36.96 & 0.336 \\
\hline Levator avulsion, $n(\%)$ & $18(25.4)$ & 0.474 \\
\hline
\end{tabular}


stage of prolapse was found (using Pearson's $\chi^{2}$ test) to be related to PR $(p=0.077)$. We further explored this concept using symmetric measures of association $(\gamma=-0.353)$, indicating that PR decreases with increasing prolapse severity. We did not find any association between parity and PR.

We found a mildly significant relationship between hiatal distance on contraction and PR $(p=0.052)$. Post-hoc testing (least significant difference and Bonferroni) was used to elucidate the relationship, and both tests found a significant difference in the mean hiatal distance on contraction between the patients who retained the pessary for $\leq 30$ days $v$. those who retained it for between 6 months and 1 year ( $p$-values of 0.016 and 0.049 , respectively). This was further explored using a $\chi^{2}$ test of the hiatal distance on contraction, and $\mathrm{PR}$, and found a significant association $(p=0.021)$. Symmetric measures testing was then used to further unpack the relationship, and it was shown that the smaller the hiatal distance on contraction, the better the PR $(\gamma=-0.439)$. We did not find any significant association between levator ani avulsion, as diagnosed on ultrasound, and PR $(p=0.474)$.

\section{Discussion}

Our study did not find any significant association between age and PR. With regard to pelvic floor muscle tone, although we were not able to comment on resting tone and PR, average oxford resting tone was not found to influence the likelihood of PR $(p=0.70)$. If one were to look at initial pessary-fitting success, most studies agree that it is not affected by any specific patient demographic (such as age, BMI, parity or pelvic floor muscle tone) or comorbidities. However, one recent study looking at the factors affecting pessary-fitting success found a decreased likelihood of success in patients of younger age, higher BMI or underactive pelvic muscle tone ${ }^{[13]}$ Focusing on PR in the short to long term, Maito et al. ${ }^{[12]}$ found PR to be inversely associated with age, specifically $>65$ years.

Our finding that maximum PR was negatively affected by prior pelvic reconstructive surgeries is in keeping with those of other studies. Other studies have also shown that prior hysterectomy or pelvic surgery negatively affects the success of initial pessary fitting. ${ }^{[8,12]}$ Future prospective studies on the subject based in our unit are planned, to further examine this relationship in more detail, i.e. using a larger sample size, and considering the relationship to individual surgery type, and ethnic variation.

We found a significant inverse relationship between maximum PR and POP-Q staging. This finding is congruous with current thinking on long-term $\mathrm{PR}$, and is in agreement with the findings of Panman et al, ${ }^{[13]}$ who found a $58 \%$ fitting success rate in patients with POP beyond the hymen, and with Maito et al. ${ }^{[12]}$ and Cheung et al. ${ }^{[16]}$ who identified a negative correlation between short- to long-term PR and advanced POP. This is of interest to the clinician who is considering conservative management of a severe prolapse, when counselling regarding failure of conservative management must play a role in the discussion with the patient.

Pelvic-floor ultrasound in urogynaecology is still in its infancy. As such, there is a paucity of studies reporting the outcomes of $\mathrm{PR}$, and levator ani morphology. Cheung et al. ${ }^{[16]}$ showed that pessary expulsion within 1 year was significantly associated with increased hiatal areas on rest, contraction and valsalva, and a higher rate of avulsion. While this study was not able to show the expected association between PR and avulsion, we were able to demonstrate a significant inverse relationship between hiatal area on contraction, and PR. This certainly bears further scrutiny in a future prospective trial.

\section{Limitations}

We acknowledge our limited sample size, and the fact that our findings are applicable to ring pessaries only. The retrospective nature of this trial did not allow for perfect patient selection or data collection, and as such, valuable data, including on patient BMI, were not available.

\section{Conclusion}

This is one of the first studies evaluating the association between $\mathrm{PR}$ and functional pelvic-floor anatomy in an SA population. It contributes to the limited SA data regarding POP and pessary use, and enables greater understanding of the factors related to PR. It will assist the clinician in improving patient selection, thereby decreasing the cost and discomfort associated with the inappropriate fitment of ring pessaries. We found significant inverse relationships between clinical factors such as PR and advanced prolapse, prior pelvic reconstructive surgery, as well as levator ani hiatal area on contraction. We did not find any association between PR and levator avulsion. A prospective study is planned to further explore this interesting topic.

\section{Acknowledgements. None.}

Author contributions. Equal contributions.

Funding. None.

Conflicts of interest. None.

1. Abdool Z, Swart P. Symptomatic pelvic organ prolapse: Experience at a tertiary urogynaecology clinic. S Afr J Obstet Gynaecol 2016;22(1):18-20. https://doi.org/10.7196/SAJOG.2016.v22i1.1038

2. Kapoor DS, Thakar R, Sultan AH, Oliver R. Conservative v. surgical management of prolapse: What dictates patient choice? Int Urogynecol J 2009;20:1157-1161. https://doi.org/10.1007/s00192 009-0930-x

3. Swift S, Woodman P, O’Boyle A, et al. Pelvic organ support study (POSST): The distribution, clinical definition and epidemiologic condition of pelvic organ support defects. Am J Obstet Gynecol 2005;192(3):795-806. https://doi.org/10.1016/j.ajog.2004.10.602

4. Mant J, Painter R, Vessey M. Epidemiology of genital prolapse: Observations from the Oxford Family Planning Association Study. Br J Obstet Gynaecol 1997;104(5):579-585.

5. Naylor AC. Hysterectomy - analysis of 2901 personally performed procedures. S Afr Med J 1994;65(7):242-245.

6. Cornacchione M, Thomas C. Review of urologic problems in senior care: From urinary retention to overactive bladder. Consult Pharm 2004;Suppl A:S1-S21. https://doi.org/10.4140/TCP.s.2004.001

7. Jelovsek JE, Maher C, Barber M. Pelvic organ prolapse. Lancet 2007;369(9566):1017-1038. https:// doi.org/10.1016/S0140-6736(07)60462-0

8. Geoffrion R, Zhang T, Lee T, Cundiff GW. Clinical characteristics associated with unsuccessful pessary-fitting outcomes. Female Pelvic Med Reconstr Surg 2013;19(6):339-345. https://doi org/10.1097/SPV.0b013e3182a26174

9. Abdool, Z. The use of vaginal pessaries by South African gynaecologists. S Afr J Obstet Gynaecol 2011;17(3):64-67.

10. Mutone MF, Terry C, Hale DS, Benson JT. Factors which influence the short-term success of pessary management of pelvic organ prolapse. Am J Obstet Gynecol 2005;193(1):89-94. https://doi. org/10.1016/j.ajog.2004.12.012

11. Clemons JL, Aguilar VC, Tillinghast TA, Jackson ND, Myers DL. Risk factors associated with an unsuccessful pessary fitting trial in women with pelvic organ prolapse. Am J Obstet Gynecol 2004;190(2):345-350. https://doi.org/10.1016/j.ajog.2003.08.034

12. Maito JM, Quam ZA, Craig E, Dannre KA, Rogers RG. Predictors of successful pessary fitting and continued use in a nurse-midwifery pessary clinic. J Midwifery Womens Health 2006;52(2):78-84. https://doi.org/10.1016/j.jmwh.2005.09.003

13. Panman CM, Weigersman M, Kollen, BJ, Burger H, Berger MY, Dekker JH. Predictors of successful pessary fitting in women with prolapse: A cross-sectional study in general practice. Int Urogyneco J 2017;28(2):307-313. https://doi.org/10.1007/s00192-016-3107-4

14. Nemeth Z, Farkas N, Farkas B. Is hysterectomy or prior reconstructive surgery associated with unsuccessful initial trial of pessary fitting in women with symptomatic pelvic organ prolapse? Int Urogynecol J 2017;28(5):757-761. https://doi.org/10.1007/s00192-016-3184-4

15. Clemons Jl, Aguilar VC, Sokol ER, Jackson ND, Myers DL. Patient characteristics that are associated with continued pessary use v. surgery after 1 year. Am J Obstet Gynecol 2004;191(1):159-164 https://doi.org/10.1016/j.ajog.2004.04.048

16. Cheung RYK, Lee LLL, Chung TKH, Chan SSC. Predictors for dislodgement of vaginal pessary within 1 year in women with pelvic organ prolapse. Maturitas 2018;108:53-57, https://do org/10.1016/j.maturitas.2017.11.008 


\section{RESEARCH}

17. Cheung RYK, Lee JHS, Lee LL, Chung KH, Chan SSC. Levator ani muscle avulsion is a risk factor for expulsion within 1 year of vaginal pessary placed for pelvic organ prolapse. Ultrasound Obstet Gynecol 2017;50(6):776-780. https://doi.org/10.1002/uog.17407

18. Dietz HP. Clinical consequences of levator trauma. Ultrasound Obstet Gynecol 2012;39:367-371. https://doi/org/10.1002/uog.11141

19. Abdool Z, Dietz HP, Lindeque BG. Prolapse symptoms are associated with abnormal functiona anatomy of the pelvic floor. Int Urogynecol J 2017;28(9):1387-1391. https://doi.org/10.1007/ s00192-017-3280-0
20. Da Roza T, Mascarenhas C, Araujo B, Trinadade V, Natal Jorge R. Oxford Grading Scale v. manometer for assessment of pelvic floor strength in nulliparous sports students. Physiotherapy 2013;99(3):207-211. https://doi.org/10.1016/j.physio.2012.05.014

Accepted 28 November 2018 